\title{
High efficiency and long lifetime OLED based on a metal-doped electron transport layer
}

\author{
Jiun-Haw Lee ${ }^{\mathrm{a}, *}$, Meng-Hsiu Wu ${ }^{\text {a }}$, Chun-Chieh Chao ${ }^{\mathrm{b}}$, \\ Hung-Lin Chen ${ }^{\mathrm{b}}$, Man-Kit Leung ${ }^{\mathrm{b}, \mathrm{c}}$ \\ ${ }^{a}$ Graduate Institute of Electro-Optical Engineering and Department of Electrical Engineering, National Taiwan University, No. 1, Sec. 4, \\ Roosevelt Road, Taipei, Taiwan, Republic of China \\ ${ }^{\mathrm{b}}$ Department of Chemistry, National Taiwan University, Taipei, Taiwan, Republic of China \\ ${ }^{\mathrm{c}}$ Institute of Polymer Science and Engineering, National Taiwan University, Taipei, Taiwan, Republic of China
}

Received 23 August 2005; in final form 22 September 2005

Available online 19 October 2005

\begin{abstract}
The OLED performance of cesium (Cs) doped 4,4'-bis(5-phenyl-[1,3,4]oxadiazol-2-yl)-2,2'-dinaphthylbiphenyl (bis-OXD), a metaldoped electron transport layer, is reported. Device lifetime increases because: (1) Cs is heavy and difficult to diffuse in an organic matrix, and (2) The host material, bis-OXD, exhibits a high glass-transition temperature $\left(T_{\mathrm{g}}\right)$ of $147^{\circ} \mathrm{C}$. The average roughness of the thin film is small hence the leakage current of the corresponding OLED devices is low. By using a silver cathode, an OLED with a $2.59 \mathrm{~V}$ reduction in driving voltage, a $47.3 \%$ increase in current efficiency, and a 3.14 times enhancement in operation lifetime was demonstrated.
\end{abstract}

(C) 2005 Elsevier B.V. All rights reserved.

\section{Introduction}

Organic light-emitting displays (OLED) have recently attracted much attention because of the advantages of lowpower consumption, high brightness, high contrast, and low cost $[1,2]$. However, high driving voltage and short operation lifetime are major limitations for the current OLED devices in applications as compared to the other competitive display technologies, such as semiconductor light-emitting diodes (LEDs) and liquid crystal displays (LCDs).

In order to break through these limitations, organic materials having high conductivity and good electrical contact with electrodes are desired. Recently, doping techniques, which have been widely used in forming inorganic semiconductors, have been applied for forming organic $\mathrm{p}$ - and n-type materials [3]. Although efficient p-type doping is possible for a variety of organic materials, examples based on the n-type doping with high stability are rare [4]. A typical n-type doping electron transport layer (ETL) consists of a low work-

\footnotetext{
* Corresponding author. Fax: +88622367 7467.

E-mail address: jhlee@cc.ee.ntu.edu.tw (J.-H. Lee).
}

function metal doped in an organic material. Co-evaporation of the materials, which is called the metal-doped (MD) technique, is commonly employed for preparing this ETL [5]. Not only the carrier injection is enhanced due to the modification at the ETL/cathode interface, but also the carrier transport is improved due to the incorporation of the metal atoms in ETL [6]. Although lithium was found to be one of the most efficient dopants, it would easily diffuse into the light-emitting layer (EML) and hence, induce the metal ion quenching effect. In a long-term operation, the current efficiency and the lifetime of the devices significantly decrease. 2,9-Dimethyl-4,7-diphenyl-1,10-phenanthrolin (BCP) and 4,7-diphenyl-1,10-phenanthroline (BPhen) are common materials for the host organic materials in MD-ETL. Their molecular structures are shown in Fig. 1. Nevertheless, their low glass-transition temperatures $\left(T_{\mathrm{g}}\right.$ ) of 83 and $62^{\circ} \mathrm{C}$ limit the operation lifetime of the devices [7]. Co-evaporating high $T_{\mathrm{g}}$ materials, such as Balq ( $T_{\mathrm{g}}$ : $92{ }^{\circ} \mathrm{C}$ ) with Li:BPhen to form a mixed ETL does increase the operation lifetime. Unfortunately, in the case, the driving voltage increased. There seems to be a tradeoff between operation lifetime and driving voltage in this system. 


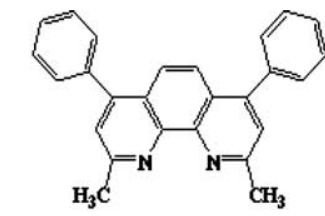

BCP

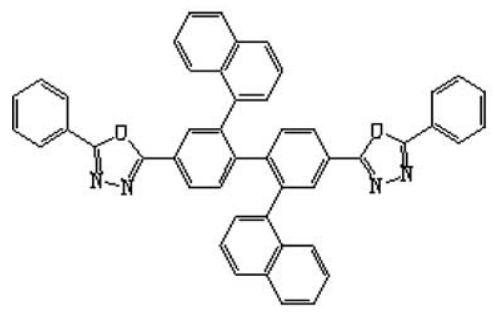

Bis-OXD

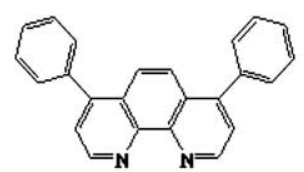

BPhen

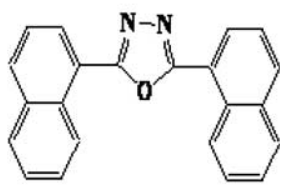

BND
Fig. 1. The chemical structure of BCP, BPhen, BND and bis-OXD.

Herein, we reported the use of cesium (Cs), a heavier alkali atom, as the dopant material in the MD-ETL. Since Cs is much heavier than $\mathrm{Li}$, we expected that the metal-atom diffusion rate would be less. However, the work function of $\mathrm{Cs}$ is smaller in comparison with $\mathrm{Li}$. Therefore, an organic material that is compatible with $\mathrm{Cs}$ and has high electron mobility is highly desired. In the present work, we adapted 4,4'-bis(5-phenyl-[1,3,4] oxadiazol-2-yl)-2,2'-dinaphthylbiphenyl (bis-OXD), shown in Fig. 1, as the host for the ETL matrix. In addition, 2,5-(binaphth-1-yl)-1,3,4-oxadiazole (BND) was employed as a model for comparison [8].

The family of oxadiazole (OXD) compounds has been extensively used as electron transporting materials [9]. We are interested in developing OLED devices based on this family for several reasons: (1) The coordination-ability of 1,3,4-oxadiazoles, including the oxygen and nitrogen atoms, with Cs should be reasonably good, allowing the Cs atoms to be dispersed evenly into the organic matrix [10]. (2) The electron mobility in OXD matrix is high [9]. (3) The lowest unoccupied molecular orbital level of OXD is close to the host of our EML, tris-(8-hydroxyquinoline) aluminum (Alq3) [11]. This makes the electron drifting from ETL to EML easier. This may also lead to a decrease in the driving voltage and an increase in the current efficiency.

In our design, we adapted the conjugated bis-oxadiazole derivatives to study. In addition, we intentionally introduced the naphthyl groups on the biphenyl backbone in order to create conformational isomerism. The conformational isomerism between aromatic rings would result in plentiful conformational isomers that reduce the chance of molecular stacking, slow down the nucleation process, and retard the rate of crystalline formation. This would be beneficial for the amorphous thin film formation. Furthermore, introducing rigid side-groups onto the bisoxadiazole would increase the chance of intermolecular entanglement and a higher $T_{\mathrm{g}}$ would be achieved. In particular, it has been reported that an oxadiazole with naphthyl substituent was found to possess high potential of electron transport [8].

The host material bis-OXD is a newly designed organic material with good thermal stability and high $T_{\mathrm{g}}$ of $147^{\circ} \mathrm{C}$. Careful study revealed that bis-OXD could form an ultra flat thin-film through thermal evaporation and deposition in high vacuum. Fig. 2 shows the atomic force microscope images of BND and bis-OXD thin films. The mean roughness of BND and bis-OXD were found to be 151.03 and $0.113 \mathrm{~nm}$, respectively. As will be shown later, the high mean roughness value of BND results in high leakage current, low current efficiency, and short operation lifetime in an OLED. Although no detailed study about the BND film has been reported in the relevant literature, we tentatively attribute the high roughness of the film surface to the

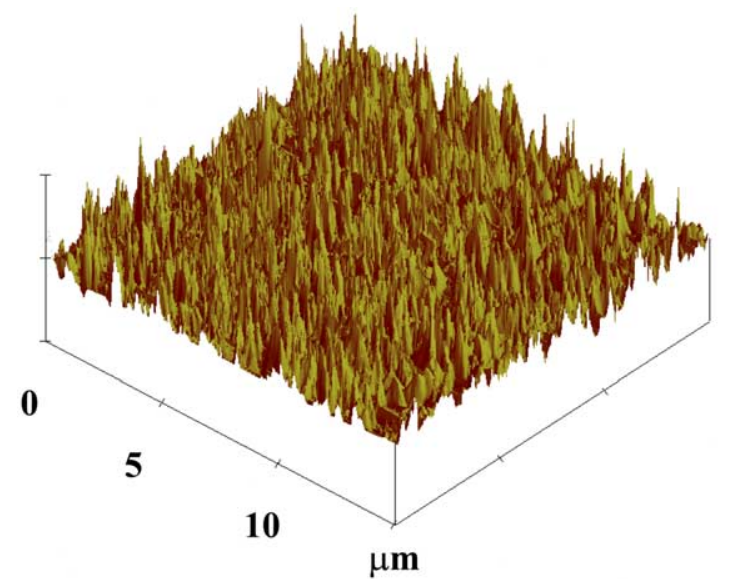

a
$Z$ axis: $1500 \mathrm{~nm} / \mathrm{div}$ Mean roughness: $151.03 \mathrm{~nm}$ Max height: $2.225 \mu \mathrm{m}$

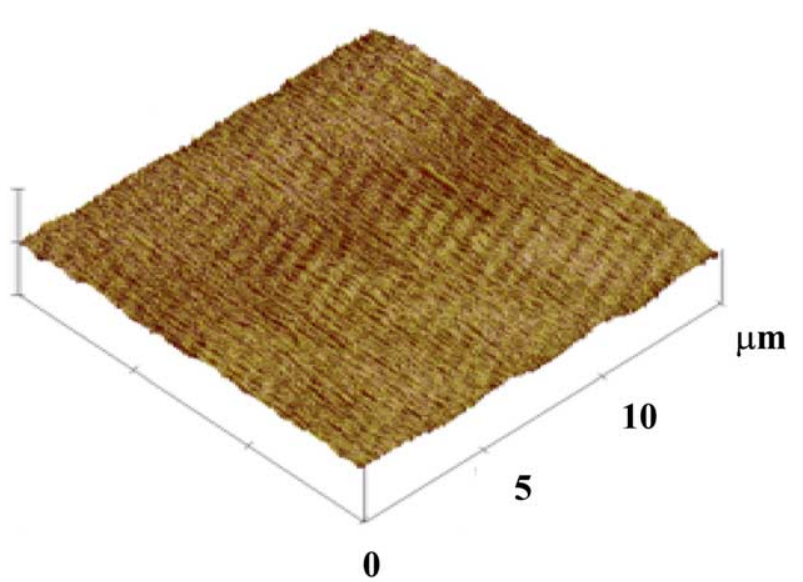

b
$Z$ axis: $5 \mathrm{~nm} / \mathrm{div}$
Mean roughness: $0.113 \mathrm{~nm}$
Max height : $1.508 \mathrm{~nm}$

Fig. 2. AFM images of (a) BND and (b) bis-OXD thin films. 
crystallization of the compound. On the other hand, due to the formation of the flat surface, the glassy bis-OXD MDETL can form ohmic contact with the cathode materials, including $\mathrm{Ag}$ and $\mathrm{Al}$, regardless of the work function. Co-evaporation of Cs and bis-OXD forms a homogeneous organic layer with low resistivity. The driving voltage of the corresponding OLED device was found to be lower in the later experiments. We will present experimental results and related discussions in Section 2 and conclude in Section 3.

\section{Results and discussion}

In all of the following experiments, we used ITO glass substrates with low sheet resistivity $(10 \mathrm{Ohm} / \mathrm{sq}$.) and flat surface roughness $\left(R_{\mathrm{a}}<1 \mathrm{~nm}\right)$. The size of the active region in our test pixel is $0.5 \times 0.5 \mathrm{~cm}$. In our devices, we used $N, N^{\prime}$-diphenyl- $N, N^{\prime}$-bis(1-napthyl)-1,1'-biphenyl-4,4'-diamine (NPB) as the hole-transport layer (HTL) material, and tris-(8-hydroxyquinoline)aluminum (Alq3) as the EML. In our control device, Alq3 was used as the ETL and $\mathrm{LiF}$ of $1.2 \mathrm{~nm}$ in thickness was used as the electron injection layer between the ETL and the Al cathode. In our MD-OLEDs, we used $\mathrm{Cs}$ as the dopant and BCP, bis-OXD or BND as the host materials of the ETL. The metal-dopant concentration, which is optimized as shown in [12], is $10 \%, 20 \%$ and $50 \%$, respectively in the molar ratio for $\mathrm{BCP}$, bis-OXD and BND. The deposition rate of the organic materials of the ETL is controlled at $0.1 \mathrm{~nm} / \mathrm{s}$ with different Cs rate. All the organic materials were sublimed before deposition. Since bis-OXD is a novel compound, the thermal properties of bis-OXD were examined by sublimation, thermogravimetric analysis (TGA), and differential scanning calorimetric analysis (DSC). The scanning rates of $10^{\circ} \mathrm{C} / \mathrm{min}$ were employed in the TGA and DSC analyses. Bis-OXD is stable during sublimation at 245 $253^{\circ} \mathrm{C}$ under the pressure of $5 \times 10^{-6}$ Torr without decomposition. On the other hand, the compound shows a $5 \%$ weight lost at $438.4{ }^{\circ} \mathrm{C}$ in TGA, indicating that the compound would be decomposed at high temperature. The compound shows a glass-transition temperature $\left(T_{\mathrm{g}}\right)$ of $147^{\circ} \mathrm{C}$ in DSC analysis. The high $T_{\mathrm{g}}$ of bis-OXD benefits for the OLED applications. The resistivity measured for Cs:BCP and Cs:bis-OXD thin films were $9.28 \times 10^{4}$ and $3.24 \times 10^{5} \mathrm{Ohm}-\mathrm{cm}$, respectively. The thickness of the $\mathrm{Al}$ and $\mathrm{Ag}$ cathode were $100 \mathrm{~nm}$. Table 1 illustrates the layer structures and the device performance. After organic and metal deposition, we encapsulated the devices in the glove box with $\mathrm{O}_{2}$ and $\mathrm{H}_{2} \mathrm{O}$ concentrations below $1 \mathrm{ppm}$. Prior to the lifetime test, devices were characterized with a Keithley 2400 source meter for current-voltage measurement and with a PR-705 photometer for the brightness measurement. In our lifetime test, we fixed the initial luminance at $10000 \mathrm{~cd} / \mathrm{m}^{2}$ and supplied a constant current to our devices. The brightness and voltage were recorded with a PR-705 photometer and a Keithley 2400 source meter at different durations.

Current density versus voltage $(J-V)$ and current efficiency versus current density $(\eta-J)$ comparison among the five devices are shown in Fig. 3a,b, respectively. Device $\mathrm{A}$ is the conventional device with the current density and the current efficiency of $0.1 \mathrm{~A} / \mathrm{cm}^{2}$ and $3.35 \mathrm{~cd} / \mathrm{A}$ at $9.72 \mathrm{~V}$ which is a typical value in such a device. When we change the ETL from Alq3 to Cs:BCP (device B), the driving voltage is reduced by $1.33 \mathrm{~V}$ and the current efficiency was increased by $4.79 \%$ at a current density of $0.1 \mathrm{~A} / \mathrm{cm}^{2}$. In an NPB/Alq3 device, holes in NPB exhibit higher mobility than electrons in Alq3 [13]. That means the electric field in ETL is higher than that in HTL. Such a carrier unbalance results in a lower recombination rate and lower current efficiency [14]. Introducing the MD technology in the device design does not only decrease the driving voltage but also improves the electron-hole balance. This explains the higher current efficiency in our MD-OLED. By replacing the $\mathrm{Al}$ cathode by an $\mathrm{Ag}$ cathode (device $\mathrm{C}$ ), the driving voltage was further reduced by $0.53 \mathrm{~V}$ and the current efficiency increased by $15.4 \%$. Since Ag has a lower optical absorption and higher electrical conductivity than $\mathrm{Al}$, such a replacement could effectively improve device performance. When we changed the host material of the EML from $\mathrm{BCP}$ to bis-OXD with an $\mathrm{Ag}$ cathode, the driving voltage and the current efficiency reach $4.93 \mathrm{~cd} / \mathrm{A}$ and $7.32 \mathrm{~V}$, respectively at the same current density. Although the conductivity of Cs:BCP is slightly higher than that of Cs:bis-OXD, the driving voltage was lower in device D than device C. The LUMO energy levels of 1.8, 2.17 and $3.7 \mathrm{eV}$ for BCP, bis-OXD and Alq3 were measured, respectively. The energy gap between $\mathrm{BCP} / \mathrm{Alq}$ is higher than that of OXD/Alq3, implying that more phonons have to be released when carriers transport from $\mathrm{BCP}$ to $\mathrm{Alq} 3$ than from OXD to Alq3. This would rationalize the higher driving voltage in device $\mathrm{C}$ as compared to device D. Further experiments for proving this assumption are ongoing.

Table 1

Layer structures and device performance of the OLEDs

\begin{tabular}{llllllll}
\hline Device & HTL $55 \mathrm{~nm}$ & EML $27.5 \mathrm{~nm}$ & ETL 27.5 nm & Cathode $100 \mathrm{~nm}$ & Driving voltage (V) & Current efficiency (cd/A) & Half-lifetime (h) \\
\hline A & NPB & Alq3 & Alq3 & LiF/Al & 9.71 & 3.35 & 13.55 \\
B & & & Cs:BCP & Al & 8.39 & 3.52 & NA \\
C & & & Cs:BCP & Ag & 7.86 & 4.16 & 17.25 \\
D & & & Cs:bis-OXD & Ag & 7.32 & 4.93 & 42.52 \\
E & & Cs:BND & Ag & 2.46 & 0.02 & NA \\
\hline
\end{tabular}

Driving voltage and current efficiency were measured at the current density of $100 \mathrm{~mA} / \mathrm{cm}^{2}$. 

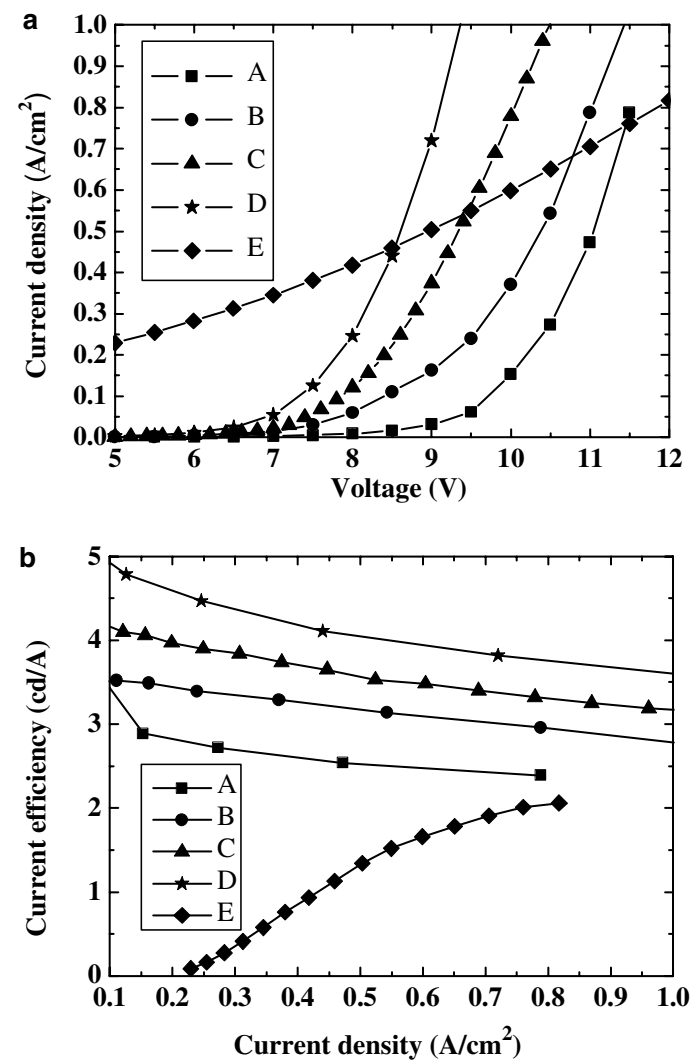

Fig. 3. Comparison of (a) current density versus voltage (b) current efficiency versus current density curves among the five devices.

The $J-V$ characteristic of device $\mathrm{E}$ is different from other devices. An ultra low turn-on voltage was observed in this case. Extrapolating the $J-V$ curve passes the origin which means such a phenomenon may result from the high leakage current of the rough BND thin film surface. The $\eta-J$ curve shows an increasing trend with an increasing current density. However, it is always the lowest among all the samples. These results indicate that the recombination rate is higher under high injection. However, the current at low injection level does not contribute to light emission. We attribute this to a leakage current.

Fig. 4 shows the results of the accelerated operation-lifetime tests of devices A, C and D. One can see that our control device, device A, has the shortest lifetime. The extrapolated half-lifetime under the brightness of $10000 \mathrm{~cd} / \mathrm{m}^{2}$ is $13.6 \mathrm{~h}$. Device B exhibits a half-lifetime of $24 \mathrm{~h}$, despite the lower $T_{\mathrm{g}}$ of the metal-dopant/host matrix. Two possible reasons may explain this phenomenon. The first is the higher power efficiency of the MD devices which generate less heat during operation. The second is the lower diffusion rate of $\mathrm{Cs}$, which is used as the dopant in the OLED devices. This leads to less quench effect. For device D containing Cs:OXD as the MD-ETL layer, the operation lifetime is as high as $42.5 \mathrm{~h}$. This is $146 \%$ and $213 \%$ longer than those for the Cs:BCP and control devices, respectively. Although the reasons behind this are unconfirmed, the high $T_{\mathrm{g}}$ characteristics of OXD may play an important role in the high efficiency and the long operation lifetime.

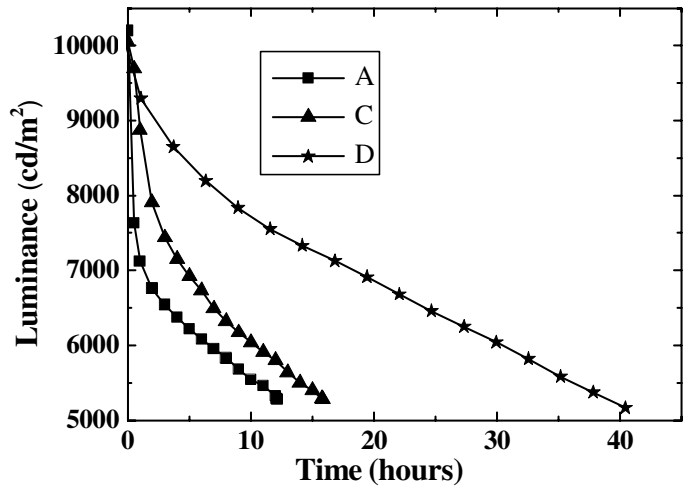

Fig. 4. Accelerated operation-lifetime measurement among devices A, C, and $\mathrm{D}$.

\section{Conclusions}

In summary, we have shown novel high performance OLED devices based on new MD-ETL and cathode materials. These could effectively decrease the driving voltage of the device and increase the current efficiency and operation lifetime. Ag was used as the cathode electrode to lower the ohmic loss and reduce the optical absorption. We chose Cs as the dopant material in the ETL to decrease the effects of metal ion diffusion, and inhibit the quenching effects in a long-term operation. For devices with MD-ETL, not only the driving voltage was decreased, higher current efficiency was also achieved due to better charge balance. Perhaps, due to the high thermal stability and good energy alignment with EML, the lifetime of the devices based on Cs doped bis-OXD ETL increased.

\section{Acknowledgement}

This work was supported by RiTdisplay Corporation.

\section{References}

[1] C.W. Tang, S.A. Vanslyke, Appl. Phys. Lett. 51 (1987) 913.

[2] C.W. Tang, S.A. Vanslyke, J. Appl. Phys. 65 (1989) 3610.

[3] J. Huang, M. Pfeiffer, A. Werner, J. Blochwitz, S. Liu, K. Leo, Appl. Phys. Lett. 80 (2002) 139.

[4] S.T. Lee, Z.Q. Gao, L.S. Hung, Appl. Phys. Lett. 75 (1999) 1404.

[5] J. Kido, T. Matsumoto, Appl. Phys. Lett. 73 (1998) 2866.

[6] T. Oyamada, H. Sasabe, C. Adachi, S. Murase, T. Tominaga, C. Maeda, Appl. Phys. Lett. 86 (2005) 033503.

[7] B.W. D'Andrade, S.R. Forrest, A.B. Chwang, Appl. Phys. Lett. 83 (2003) 3858.

[8] H. Tokuhisa, M. Era, T. Tsutsui, S. Saito, Appl. Phys. Lett. 66 (1995) 3433.

[9] S. Oyston, C. Wang, G. Hughes, A.S. Batsanov, I.F. Perepichka, M.R. Bryce, J.H. Ahn, C. Pearson, M.C. Petty, J. Mater. Chem. 15(2005) 194.

[10] J.F. Wang, G.E. Jabbour, E.A. Mash, J. Anderson, Y. Zhang, P.A. Lee, N.R. Armstrong, N. Peyghambarian, B. Kippelen, Adv. Mater. 11 (1999) 1266.

[11] Y. Kawabe, J. Abe, Appl. Phys. Lett. 81 (2002) 493.

[12] J.H. Lee, M.H. Wu, C.C. Shiau, in: Taiwan Display Conferences, Taipei, Taiwan, June 10-11, 2004.

[13] W. Brütting, S. Berleb, A.G. Mückl, Org. Electron. 2 (2001) 1.

[14] Y. Qiu, Y. Gao, P. Wei, Liduo Wang, Appl. Phys. Lett. 80 (2002) 2628. 